\title{
ESCRITURAS INÉDITAS DE OSEIRA ANTERIORES A 1435
}

\author{
MIGUEL ROMANÍ MARTÍNEZ \\ PABLO S. OTERO PIÑEYRO MASEDA \\ Universidad de Santiago \\ CSIC-Xunta de Galicia \\ Instituto de Estudios Gallegos "Padre Sarmiento"
}

\section{Resumen}

Se editan nueve documentos monásticos inéditos pertenecientes al fondo de Oseira, pero no incluídos en su momento en la colección diplomática de este monasterio.

\section{Palabras clave}

Paleografía, monasterio, Edad Media, Oseira, colección diplomática.
Abstract

Nine unpublished documents belonging to the collection of Oseira's monastery are edited. However, they were not included in a diplomatic collection of this same monastery at first.

\section{Keywords}

Paleography, Monastery, Middle Ages, Oseira, diplomatic collection. 
La publicación de fuentes documentales gallegas debe ser un continuo empeño de paleógrafos y diplomatistas; las líneas que siguen tienen por objeto dar a conocer nueve documentos del primitivo fondo de pergaminos ursariense que en su momento no pudieron incluirse en la Colección Diplomática de Oseira ${ }^{1}$ : ahora se trata de completar la colección de pergaminos ursariense editándose por orden cronológico.

Con estos nueve documentos se completa, hasta el momento, la colección de pergaminos ursariense desde el año 1015 a los inicios del año 1435. Su edición se ofrece por orden cronológico.

Tres piezas (docs. núms. 1, 3 y 4) proceden del fondo de pergaminos del Archivo de la Real Chancillería de Valladolid, seguramente por haberse enviado a dicho organismo a causa de un pleito de Oseira sobre unos bienes en Villamarín (Orense) con la familia de los Novoa ${ }^{2}$.

El documento núm. 2 es una bula del Papa Bonifacio VIII, datada en 1302, concediendo la exención del pago del diezmo de las tierras cultas e incultas a la orden cisterciense.

Los núms. 6 y 7 se conservan gracias a que están insertos en otro documento copiado en el conocido como "Tumbo D" del archivo de la catedral compostelana; en este caso se trata de una donación otorgada en 1391 por Pedro Martínez, do Outeiro, y su hijo Estevo Pérez, clérigo, de tres partes iguales del lugar do Outeiro, parroquia de San Martiño de Cameixa, a los monasterios de Santa María de Aciveiro (Pontevedra), Santa María de Oseira y al cabildo y mesa capitular de la Catedral de Santiago, a la que sigue el testamento del mismo clérigo Estevo Pérez, por el que de nuevo dona en tres partes iguales todos los bienes que posee en tal lugar do Outeiro a los citados monasterios y mesa capitular compostelana.

Los tres restantes (núms. 5, 8 y 9) proceden del Archivo Catedralicio de Orense. Uno de ellos es práctimente ilegible; otro no se pudo localizar en el archivo ${ }^{3}$,

\footnotetext{
${ }^{1}$ Miguel Romaní Martínez, Colección Diplomática do Mosteiro Cisterciense de Sta. María de Oseira (Ourense), 1025-1310, Santiago, Tórculo; Dpto. de Historia I de la Universidad de Santiago, 1989, 2 vols; Miguel Romaní Martínez, et al., Colección Diplomática do Mosteiro Cisterciense de Sta. María de Oseira (Ourense), 1310-1399, Santiago, Tórculo; Dpto. de Historia I de la Universidad de Santiago, 1993; y Miguel Romaní Martínez, et al., Colección Diplomática do Mosteiro Cisterciense de Sta. María de Oseira (Ourense), 1400-1435, Santiago, Tórculo; Dpto. de Historia I de la Universidad de Santiago, 2003.

${ }^{2}$ Importante linaje orensano que ya desde el siglo XIII se vinculan a la tenencia de la terra de Aguiar. Alguna información de interés sobre este viejo linaje gallego en Olga GALLEGo DomínguEZ y Francisco FARIÑA BUSTO, "Os Nóboa, unha linaxe ourensá", Boletín auriense, 35 (2005), 155-176. Un completo panorama nobiliario de la Galicia de la Edad Media en Eduardo PARDo DE Guevara Y VALDÉs, Los señores de Galicia. Tenentes y condes de Lemos en la Edad Media, A Coruña, Fundación Pedro Barrié de la Maza, 2000 (Galicia Histórica).
}

${ }^{3}$ Doc. núm. 8. 
por lo que sólo se han podido tomar los datos del documento extractado en el Repertorio para las escripturas antiguas del Archivo Bajo ${ }^{4}$. El último, del que sólo se conserva el extracto del citado Repertorio, está mal datado, ya que el abad otorgante, frey Gómez, ejerció su abadengo entre 1424 y los inicios de $1435^{5}$, y en 1437, año que le asigna el Repertorio, ya regía el monasterio de Oseira desde algo más de dos años el abad don frey Juan de Sante.

\section{SIGLAS Y ABREVIATURAS UTILIZADAS}

Para ofrecer una mayor claridad, y aunque ya sean de sobra conocidas, a continuación se citan las más habituales:

AC- Archivo Catedralicio.

AHN- Archivo Histórico Nacional.

ARChV- Archivo de la Real Chancillería de Valladolid.

Col. Perg.- Colección de pergaminos.

doc.- documento.

extr.- extracto.

orig.- original.

pág. o págs.- página o páginas.

part.- partida.

perg.- pergamino.

v.- véase o véanse.

\footnotetext{
${ }^{4} \mathrm{M}^{\mathrm{a}}$ José Portela, et al., (Ed.), Repertorio para las escripturas antiguas del Archivo Bajo: Catálogo del Archivo Monacal de Oseira en 1629, [Santiago de Compostela], Tórculo, 1993.

${ }^{5} \mathrm{El}$ abadiazgo de este frey Gómez de Ambasmestas parece que se interrumpió brevemente durante el año 1430, v. Miguel Romaní MARTínez, "Notas sobre el monasterio de Oseira, abadologio y jerarquías entre 1414-XII-24 y 1435-IV-20", en Miguel Romaní Martínez y Ma Angeles Novoa Gómez (eds.), Homenaje a José García Oro. Facultade de Xeografía e Historia, Santiago de Compostela, Universidade de Santiago de Compostela, Servicio de Publicacións e Intercambio Científico, 2002, 308-313.
} 


\section{FUENTES MANUSCRITAS UTILIZADAS}

\section{ARCHIVO DE LA CATEDRAL DE ORENSE}

I.1. Pergaminos Monásticos. Monasterio de Oseira.

Signaturas: 4332, 4677

I.2. Fondo de Códices.

Repertorio para las escripturas del Archivo Bajo.

Es un catálogo de documentos del monasterio de Oseira, escrito en 1629;

los documentos se ofrecen en extracto.

\section{ARCHIVO HISTÓRICO NACIONAL}

II.1. Sección Clero (Fondo Monástico).

Serie Pergaminos. Monasterio de Osera.

Carpetas: $1553, n^{\circ} .6 ; 1553 n^{\circ} .7$.

II.2. Sección Códices.

Monasterio de Osera.

Libro Tumbo de Pergamino.

1008-B, fols. $1 \mathrm{r}-1 \mathrm{v}$ y fol. $63 \mathrm{v}$.

\section{ARCHIVO DE LA CATEDRAL DE SANTIAGO}

III.1. Índice General de Legajos.

Legajo no. 5 (Tumbo D), fols. 38r.- 40r.

\section{ARCHIVO DE LA REAL CHANCILLERÍA DE VALLADOLID}

IV.1. Colección de Pergaminos

Carpetas:

$11, \mathrm{n}^{\mathrm{o}} .12,13,14$. 


\section{CATÁLOGOS Y BIBLIOGRAFÍA CITADA EN LAS FICHAS CATALOGRÁFICAS}

- LeIrós FERnÁndez, Emilio, Catálogo de los pergaminos monacales del Archivo de la S. I. Catedral de Orense, Santiago, El Eco Franciscano; Dirección General de Archivos y Bibliotecas, 1951 (Bibliotecas y Archivos Eclesíasticos, 1).

- Portela, María José; Garrido, Margarita y Miguel Romaní, Repertorio para las escripturas del Archivo Bajo. Catálogo del Archivo Monacal de Oseira en 1629, [Santiago], Tórculo, D.L. 1993.

- Romaní Martínez, Miguel y María del Pilar Rodríguez SuÁrez, Libro Tumbo de Pergamino, un códice medieval del monasterio de Oseira, Santiago, Tórculo; Dpto. de Historia I de la Universidad de Santiago, 2003.

\section{CRITERIOS DE TRANSCRIPCIÓN}

Se utilizan las normas habituales sobre abreviaturas y abreviaciones; además, debe tenerse en cuenta lo que sigue:

- Los signos notariales se señalan en mayúsculas entre paréntesis (SIGNO).

- El fin de renglón en el original se indica con I en los docs. 1 y 3.

- Las frases o palabras escritas entre renglones se transcriben en letra normal entre $\backslash \quad /$.

- La restitución cierta de lo escrito cuando hay una laguna en el texto se escribe entre corchetes [ ].

- Lagunas no restituíbles en el texto se indican con puntos suspensivos entre corchetes $[\ldots]$.

- Las grafías aberrantes o destacables se señalan con un ( sic), que afecta a las letras o palabra/s inmediatamente anterior/es en cursiva.

- Los caracteres no presentes en el texto, pero indispensables para su sentido se transcriben entre ángulos $<>$.

- Las palabras o frases dudosas se transcriben en cursiva, seguidas de un interrogante: mel?, salvo indicación específica al contrario.

- Entre paréntesis, a la izquierda de la data, sobre el regesto, se indica, cuando es posible, la mayordomía a la que pertenece cada documento, siguiendo el Repertorio de 1629. 


\section{EDICIÓN}

\section{1}

1299, julio, 22.

El abad frey Miguel concede a Juan Gómez y a su mujer María Vázquez un casal en Villamarín, en el lugar de Figueiredo, y otro en Biduedo; estos entregan al abad un casal en Fondo de Vila de Villamarín y otro en Biduedo, recibiendo aquellos el usufructo de los cuatro lugares.

VALLADOLID, ARChV, Col. Perg., 11/12, carta part. por a.b.c., perg., orig., gallego, letra gótica cursiva, 300x140 mm.

Era de mill et CCC et XXX et VII anos, et vinte et dous dias I do mes de juyo. Sabeam quantos esta carta virem, conmo I eu, Johan Gomez, con mia moller Maria Vaasquez, reçebelmos de vos don frey Migell, abbade d'Osseyra I et do convento desse meesmo lugar, en enplazamento I dous vossos cassares: un cassal en Villamarin, en I que mora Johan Lopez, en o lugar a que chaman Figeylredo, et outro en a villa que chaman Bidoedo, en que mora I Domingo Perez, por tal preyto que os tenamos de vos et I por vos en nosa vida polo ussu dos froytos I et tenamos as cassas delles en boa revor, et refalgamos se mester for. Et por estes dous cassares I que de vos don abade et convento reçebemos, dalmos a vos sobreditos logo en presente, outros dous I cassares: um delles en Villamarin, no lugar a que I chaman Fondo de Villa, et outro en a villa de Bidoedo, I con sas pertenças a monte et a fonte. Et deste dia I endeante tollemolos de nossu pod<e>rio et metolllos no vosso, que façades delles toda vosa I voentade.

Et se non [...] vossa I parte ou doutra contra este nosso enplazamento que I nos fazemos quiser viir, peyte a vos mill moravedis I de boa moeda, et a a voz del rey outros tantos; I et o nosso enplazamento fique senpre en sua relvor et firme.

Os quaes cassares reçebemos I logo de vos, que os tenamos de vos et por vos en I nossa vida, pollo ussu dos froytos con estes outros I dous de susoditos que de vos reçebemos, os I quaes cassares todos quatro non devemos apalrar mal nen enallear do jur do moesteyro, I su a pena sobredita dos mill moravedis; mays oultorgamos et prometemos que fiquem todos IIII $^{\circ}$ livres I et quitos a o moesteyro a morte de nos anbos por erdade para senpre, et seiades senpre con elles anparadlos por nos et por nossos beens. 
Et que esto seia mais I firme, nos as partes sobreditas, mandamos a Fralnçisco Peres, notario del rey en Castella et en Bulval et en o Bollo de Senda, que faça desto un estrulmento partido por a.b.c.; et eu, don Migell, abade I sobredito, outorgo por min et pollo convento esta I carta en todo segundo en ella dis.

Ts.: Fraly Johan et frey Diego, et frey Migell, monges d'Osseira, I frey Estevo de San Lourenço, Roy F., de Faro, Johan Peres, I [...] Johan Perez, da Regeira, Johan Peres $[\ldots]$.

[A..B..C.]

1302, diciembre, 18.- Letrán.

El Papa Bonifacio VIII concede la exención del pago del diezmo de las tierras cultas e incultas a la Orden Cisterciense.

Inserto en MADRID, $A H N, 1553 / 6$, perg., orig., de fecha 1440, diciembre, 22, Florencia; también a su vez inserto en MADRID, $A H N, 1553 / 7$, copia autorizada por Estevo Fernández de Camporramiro, canónigo y notario público apostólico de la iglesia de Orense, realizada en 1478, marzo, 11, Orense.

MADRID, AHN, Serie "Códices", n. ${ }^{\circ} 1008$ B (Tombo Vello de Oseira), fols. 1v-2r, copia simple, latín, letra cortesana.

Edita Romaní MARTínez et al., Libro Tumbo..., págs. 5-6.

Bonifacius episcopus servus servorum Dei. Dilectis filiis universis abbatibus abbatissis et conventibus ordinis cisterciensis tam presentibus quam futuris, salutem et apostolicam benedictionem. In ecclesie firmamento vester ordo nitore claro corruscans universalem gregis dominia aulam illuminat et currentibus in stadio rectum iter insinuat quo ad salutis vravium facilius pervenitur nos quidem ob hoc et propter magne devotionis affectum, quam ad nos et apostolicam sedem habetis ordinem ipsum ac nos et alios eiusdem ordinis professores intima caritate prosequimur ac sinceris affectibus excitamur ad vestra et illorum conmoda, in quibus honeste possumus promovenda. Ideoque premissorum intuitu et obtentu dilecti filii nostri Roberti tituli Sancte Pudenciae presbiteri cardinalis qui tanquam prefati ordinis quem professus existit, promotor assiduus necessitates vestras et dicti ordinis nobis reverenter exposuit et super illis vestre provisionis auxilium imploravit vobis, auctoritate presentium indulgemus ut de terris vestris cultis et incultis ad ordinem vestrum expectantibus, quas aliis concessistis vel concedetis im posterum excolendas, de quibus tamen aliquis 
decimas seu primitias non percepit nullus a vobis seu cultoribus terrarum ipsarum aut quibuscumque aliis decimas seu primitias exigere vel extorquere presumat. Nos enim nihilominus irritum decernimus et inane quicquid contratenorem huius modi indulgencie fuerit attemtatum. Nulli ergo omnino hominum liceat hanc paginam nostre concessionis et constitutionis infringere vel ei ausu temerario contraire.

Si quis autem hoc attemptare presumpserit, indignationem omnipotentis Dei et beatorum Petri et Pauli apostolorum eius se noverit incursurum.

Datum Laterani XV kalendas ianuarii pontificatus nostri anno octavo.

1313, agosto, 10.

El abad frey Pedro y María Eanes se avienen en la disputa sobre dos casares en Villamarín, los cuales aquella alegaba que su padre, el escudero Juan Pérez, había malvendido al monasterio.

VALLADOLID, ARChV, Col. Perg., 11/13, carta part. por a.b.c., perg., orig., gallego, letra gótica cursiva, $270 \times 175 \mathrm{~mm}$.

A..B..C.- Era de mill et CCC et LI anno, X dias andados d'agosto. Cunusçuda coussa sega a quantos esta carta viren, I conmo fose contenda en juyzo por ante Affonso Viviançe, vigario do honrrado padre et sennor don Gonçalo, pola graça de I Deus, bispo d'Ourense, ontre don frey Pedro, abbade d'Osseyra, et o convento desse lugar, da huna parte, et Maria Eanes, I que se dizia heree de Johan Perez, scudeyro de Villamarin, et Johan Ares, seu marido, da outra parte, sobre raçon de dous calsares que stan na dita villa de Villamarin, hun delles, sta no luguar que chaman Barreo, et o outro no lugar I que chaman Fondo de Villa, os quaes cassares o abbade et o convento dizian que lles pertiiçian por raçon que os conlpraran do dito Johan Perez.

Et Maria Anes dizia que como quer que os abbade et convento conprassen, que o dito Johan Perez I fora enganado de mays qua meatade do justo preço.

Et as partes de seu prazer et de sua voontade, I et por mandado do dito vigario, a tal aviinça et conpossiçon veeron, conben a saber: que o dito abbade et convenlto den logo en presente en sua vida de Maria Eanes et de Johan Ares, seu marido, tan sollamente en voz et I en nome do dito moosteyro por lo ussu dos froytos o seu cassar de Barreo, o qual cassar delles tevo dolnna Maria Fernandez Coronel, a 
monte con todas suas perteenças, o qual cassar e en essa villa de Villamarin, no qual I morou Stevo Perez, por tal preyto que o lavren et paren ben, que non fallesca per myngoa de lavor et de boa parança, I et mantenna as cassas et as arvores del en boa revor et as refaçan se mester for, et den del cada anno I de conosçença a o dito moosteyro por dia de Santa Maria d'agosto hun quarteyro de boo çenteo en salvo na sua I villa de Touges por la tulla d'Alquirime, por (sic) dan as outras dereyturas.

Et por esta aviinça et graça que fazen I a dita Maria Anes et a seu marido Johan Ares, essa Maria Eanes con seu marido Johan Ares, a vos presentes et oultorgantes, quitan et renunçian para por si et por toda sa voz, a o dito abbade et convento d'Osseyra et a todos seus suçessores toda demanda et dereyto et auçon, que ella a, ou de dereyto, poderia aver I contra o dito moosteyro sobre razon dos ditos cassales de Barreo et de Fondo de Villa que foron do I dito Johan Perez, escudeyro, tanben de demanda que a dita Maria Anes fazia por razon de enganno que dizia I que o dito Johan Perez fora enganado como por outra qualquer maneyra ou demanda que sega.

Et a molrte do postremeyro delles, o moosteyro por sua propia autoriade possa entrar et reçeber o dito cassar I con quantas boas paranças elles y fezeren, salvo gaando ou pan coll<ey>to que soya a dita Maria Anes ou Johan Ares I trouxeeren ou teveren, que o agan.

Et se algen da sa parte delles ou doutra, contra esta aviinça ou conpolçisson quisser passar, peyte a outra parte mille maravedis da boa moneda de pena, et esta carta stia senpre en relvor, et cada huna parte sega anparada por los beens da outra gaanados et por gaanar, assi mel? I como feyta?

Et que sega firme et stavel ontre si, fazen esta carta partida por a.b.c., que a parte que a non agardar, peyte a outra parte a pena sobredita.

Et esto foron presentes et outorgantes polo moosteyro I por don abade frey Lourenço, prior d'Osseyra, et frey Rodrigo, çellareyro et procurador do dito moosteyro por carta do sennor I abbade, et frey Garçia, meestre, et frey Lourenço, stalleyro, et frey Gillelme, enfermeyro, et frey Afonso, I tessoureyro, et o outro convento con elles.

Ts. que steveron presentes: Johan Martinez, de Barreo de Villamarin, I et Johan Perez, de Piellas, et Martin Monteyro, da enfermaria, et Pedro Domingues, dito Foreyro, et Johan Martines, home I de don abbade.

Et eu, Johan Fernandez, notario por Domingo Martinez, notario publico del rey, a esto I foy presente, et por mandado das partes esta carta scrivy, et pus en ella meu sinal, I semellante o do dito Domingo Martinez, que tal est (SIGNO). 
1333, agosto, 16.- Oseira.

El abad frey Alfonso afora a Pedro Anes, a su mujer Teresa Pérez y a una voz el casal de Malburgo, que fue de Juan Pérez de Novoa, feligresía de Santiago de Villamarín, por pago de la mitad del grano y del lino, ocho libras de blancos por foros, y otras prestaciones.

VALLADOLID, ARChV, Col. Perg., 11/14, carta part. por a.b.c., perg., orig., gallego, letra gótica cursiva, $160 \times 285 \mathrm{~mm}$.

A..B..C.- Sabean quantos esta carta viren, como nos frey Afonso, abbade d'Oseyra et convento desse meesmo lugar, damos a vos, Per'Anes, et a vossa moller Tareysa Perez, et a huna voz qual nomear a postrimeiro de vos a sua morte, que seia semelavel de vos, de que nos possamos aver os nossos dereytos en paz a teer de nos et por nosso moesteyro en vossa vida, tam solamente pollo huuso dos froitos, ho nosso casar de Malburgo, que foy de Johan Perez de Novoa, o qual jax (sic) su sino de Santiago de Villamarim, a montes et a fontes, con todas suas perteeças, por tal preyto que o lavredes et o paredes bem, en gissa que se non percam os froitos del per mingoa de lavor et de boa parança, et tennades as casas senpre en boa revor, et as refaçades se mester for, et moralla edes per (sic) vos; et daredes del cada aanno (sic) meo de quanto graao Deus y der per noso moordomo, et meo de linno; et proveeredes o mordomo de comer et de bever mentre coler convosco os froitos do dito lugar; et darnos edes cada ano por dia de Natal oito libras de blancos por foros da moeda del rey dom Alfonso, ou a contia delles. Et faredes del os outros serviços que senpre del fezerom os outros que y morarom. Et trageredes per vos et per vossa custa o nosso quinon dos froitos ha nosa tulla de Touves. Et seredes nossos vassallos mandados et obedientes. Et non tomaredes amadigo nen sennorio contra nossa voantade. Et non seredes poderossos nen na voz que ficar apus vos de venderdes nen sopinnorades, nen allenaedes o dito lugar sem mandado do moesteyro a nenguun.

Et que esto seia çerto et non venna en dulta, façemos convosco esta carta partida per a.b.c., et que a parte que a non agardar, que peyte a outra parte $\mathrm{C}$ moravedis de pena da dita moeda, et a carta fique en sua revor.

Et nos, Per'Anes et a dita Tareysa Perez, outorgamos a agardar as ditas condiçoens su a pena sobredita.

Feyta a carta en Osseyra, XVI dias andados do mes d'agosto, era de mil et CCC et LXXI anno. 
Ts. que presentes forom: Gonçalo Gil de Llamas, Afonso Nunez et Rodrig'Alvarez, escudeyros de Castrello, Sancho Garçia, de Sever, Per'Anes, home de dom abbade, et outros moytos.

\section{5}

[1362].- Oseira.

(Piedrafita)

El abad frey Domingo afora a Juan de Deus [clérigo, capellán de la iglesia de Pedrafita] y a una voz el casal de [Otero], por pago de [seis cuarteros de pan].

ORENSE, $A C$, n. ${ }^{\circ}$ 4332, carta part. por a.b.c., perg., orig., gallego, letra gótica documental, 270x130 mm., prácticamente ilegible.- Extr., Repertorio, pág. 112.

Catálogo: LeIRós, Catálogo, 4332.

Observaciones: Las frases entre [ ] del regesto se toman del Repertorio, del cual se toma también la fecha, ya que en el Catálogo de LEIRós se data erroneamente en 1450.

A..B..C.- [Sabeam quantos esta carta viren como nos], frey Domingo, abbade d'Oseyra, et o convento desse lugar, damos a vos Johan de Deus [...] et a huna voz qual nomee o postremeyro de vos a vosa morte [...] de nos et do noso moesteyro, en vossa vida de todos tres, tan solamente por lo huso dos froytos [...], o noso casar [...] con todas suas perteeças, por tal pleyto et condiçon que seades nosos vasallos mandados et obedientes [et lavraredes et pararedes bem o dito casar como se non percan os] froytos del por mingoa de lavor et de boa parança, et tenades as casas del [...] nos dedes del cada anno o sesto en todo o mes de [...] en a nosa tulla de [...] seis quarteyros de pan en duas terços de çenteo a hun de [...] et este casar non venderedes nen deitaredes [...] sen noso mandado, nen tomaredes y amadego nen senorio [...].

Et que esto sea çerto, fazemos convosco esta carta [...] peite çem maravedis de pena, et a carta estea en sua revor [...] sobreditas, outorgo esta carta et as [...] que o moesteyro possa tomar seu foro $[\ldots]$ as maas paranças que $[\ldots]$

Ts. frey Fernando d’Asma, Domingo [...], escripvi esta [...], et soo ts.

\section{6}

1391, marzo, 14.- San Juan de Laxas.

(Mato)

Pedro Martínez do Outeiro y su hijo Estevo Pérez, clérigo, donan tres partes iguales del lugar do Outeiro, en la parroquia de San Martiño de Cameixa, a los 
monasterios de Santa María de Aciveiro, Santa María de Oseira y al cabildo y mesa capitular de la Catedral de Santiago.

Inserto en fols. 38v.-39r de SANTIAGO, AC, Índice General de Legajos, leg. 5 (Tumbo D), de fecha 1447, julio, 28, fols. 38r-40r, copia simple.

Fol. 38v. Anno do nasçemento do noso Sennor Jhesu Christo de mill et tresentos et noveenta et huun annos, quatorse dias do mes de março. Sabeam todos que eu, Pedro Martines, do Outeyro, morador en a fregisia de San Martinno de Cameyja, et Estevoo Peres, clerigo, seu fillo, que he en terra de d'Orzellon, nos anbos en senbra, avendo devoçon en a Virgen Santa Maria, madre de noso Sennor Jhesu Christo et en o apostolo sennor Santiago, et en as vertudes et miragles que fasta aqui ha demostrados et de cada dia demostra asy por mar como por terra, et porque nos outrosy ajamos parte et quinon, nos et aqueles, donde vieron et deçenderon aqueles que nos somos tiudos et obligados en as misas et oras et novesarios et en os outros ofiçios devinaes que cada dia se disen et resan en as iglesias da Virgen Maria et en a see cathedral de Santiago, et por ende eu, o dito Pedro Martines, do Outeyro, et Estevoo Peres, clerigo, seu fillo, en a mellor forma et maneyra que podemos et devemos de dereyto, non costrengidos por força nen endozidos por enganno, mays de nosas propias et libres voontades, damos et outorgamos en pura et justa et libre doaçon inrrevocabile para senpre, asy como doaçon mellor, pode et deve valer et (tachado) de dereyto d'oje este dito dia endeante para todo senpre a Santa Maria d'Aziveyro, et convento et cabidoo, et a Santa Maria d'Oseira, et convento et cabidoo, et a o dean et cabidoo et mesa capitular et personas et benefiçiados da dita iglesia cathedral de Santiago, et a todos seus subçesores; conven a saber, a terça parte entregamente a Santa Maria d’Aziveyro, et a outra terça parte a o moesteyro et convento de Santa Maria d'Oseira, et a outra terça parte a o dean et cabidoo et mesa capitular et personas et benefiçiados da dita iglesia cathedral de Santiago, et a todos seus subçesores, a terça parte entregamente de todas las herdades, casas et casares et chantados et vinnas et lagares et cubas, con suas perteencas et dereytos que a nos perteençen en o dito lugar do Outeyro, et en toda a fregisia de San Martino de Cameija, por parte et herança de nosos padres et madres et avoos et por conpras, et por outros qualesquer rasoons et titolos, adonde et ondequer que vaan, a montes et a fontes, en a dita fregisia, con esta condiçon: que nos, en todas nosas vidas, comeamos ( $\mathrm{sic}$ ) et ajamos et levemos ho uso et froyto das ditas herdades et dos ditos beens que lles asy damos, porque nos mantenamos por eles, et que a o tenpo dos nosos finamentos que os dito $<\mathrm{s}>$ beens et herdamentos et vinnas segundo de suso son declaradas et contiudas que fiquen libres et quitos et desenbargados a o dito moesteyro et convento de Santa Maria d’Aziveyro, et a o dito moesteyro et con- 
vento de Santa Maria d'Oseyra, et a o dito cabidoo et mesa capitular, et personas et benefiçiados del sen noso enbargo, et de nosas voses et herdeyros, et todo jur et sennorio et posison et propiedade, et vos et abçon et dereyto que nos en as sobreditas herdades de todos los ditos beens que asy damos a o dito moesteyro et convento de Santa Maria d'Aziveyro et a o dito moesteyro de (tachado) et convento de Santa Maria d'Oseyra et a o dito cabidoo et mesa capitular en esta doaçon, et por ela de nos, et de nosas voses et herdeyros, o quitamos et partimos et tiramos, et en os ditos moesteyros et conventos et cabidoo et mesa capitular et personas et benefiçiandos del, o poemos, çedemos et traspasamos por esta presente carta de doaçon que das ditas herdades et beens lles fasemos, et quiremos et outorgamos des $<$ de $>$ o dia dos nosos finamentos endiante, façan delo et de cada cousa et parte delo o que quiseren et por ben toveren, asy como de suas cousas propias, quitas et libres et desenbargadas, et damoslles poder que por sy ou por quen quiseren et por sua propia abtoridade et sen mandado de juis nen de allcalde nen de outro ofiçial, posan logo de presente por vertude desta carta tomar et entrar, et tomen et entren et reçeban por sy ou por quen quiseren en seu nome, o jur et posison et propiedade real et abtual et corporal de todos los ditos beens et herdades de suso declaradas que lles asy damos en a dita doaçon, sen noso enbargo et d'outro alguno en noso nome, o qual dito jur et propiedade et posison de todos los ditos beens et cousas lles damos et en eles o poemos et traspasamos et os apoderamos en elo por esta presente carta de doaçon. Et queremos et outorgamos et nos plase que se de aqui endiante nos toveremos et usaremos os ditos beens et herdamentos suso declarados et doados, confesamos et outorgamos que os teemos et posuymos et terremos et posoyremos en as ditas nosas vidas por los ditos nosos (tachado) moesteyros et conventos et por lo dito cabidoo et personas del et de suas maaos jur et posison, et non por outro alguno. Et por mayor abundamento de dereyto, costituymonos et fasemonos poseedores et seer poseedores en eles por los ditos moesteyros et conventos et por lo dito cabidoo et mesa capitular, et personas et benefeçiados del, et non por outro alguno. Et por mays çertidue desto et garda dos ditos moesteyros et conventos deles, et do dito cabidoo et mesa capitular et personas et benefiçiados del, declaramos et disimos de todos los ditos beens et vinnas et herdades, asy lles damos en a dita doaçon por nos, non son dados nen vendidos nen traspasados nen enalleados nen concanbeados nen obligados nen enpennados en outra persona alguna, et prometemos et outorgamos que por nos et por nosas vozes et herdeyros que vos los façemos saaos et de pas a todo tenpo. /Fol.39r. Et a qual dita (tachado) \doaçon/ que asy fasemos do que sobredito he, de nosas propias et libres voontades, prometemos et outorgamos de non revocar en nosas vidas nen a tenpo de nosos finamentos por mandas nen codiçilos nen por outra escriptura alguna, et se o feseremos, que non valla nen viiremos nen pasaremos contra ela por nos nen por outro en 
nosos nomes et lugar, en juiso nen en fora de juiso, por juramento que ende fasemos a os Santos Avangeus, et en esta signal de crus + en presença deste notario et ts. de juso escriptas, et so pena de dous mill froliins de conto et de peso do qunno d'Aragon, que peytemos por pena, nos ou nosas voses et herdeyros, a os ditos moesteyros et conventos et cabidoo et personas del se contra elo formos ou pasarmos et o asy non tovermos et conprirmos; et do qual dito juramento que ende fasemos, prometemos et outorgamos de non pidir absoliçon nen relaxaçon a noso sennor o Papa nen a seu nunçio apostolico nen a arçobispo nen a bispo nen a outro prelado alguno, aynda que poderio et jurdiçon ajan de nos absolver. Et posto que a peçamos ou ajamos, prometemos et outorgamos de non usar dela. Et se o contrario feseremos, que fiquemos por elo infames et fe perjuros et que todavia sejamos tiudos et obrigados de conprir et aguardar esta dita doaçon et cousas en ela contiudas et declaradas. Para o qual todo asy teermos et conprirmos et agardarmos et pagarmos a dita pena se en ela encorrermos, obligamosnos nos et todos nosos beens moveles et raises avidos et por aver; et sçobre esto que dito he, renunçiamos et partimos de nos todas las leys et dereytos canonicos et çeviins, inperiaais et reaais et usos et custumes et liberdades que en contrario desto posamos ou poderiarmos por nos ou por outro diser ou alegar, et queremos que non vallan en juiso nen fora del nen sejamos çertos delo, oydos nen reçebidos, et en espiçial renunçiamos a ley o dereyto que dis que general renunçiaçon non valla, que nos seendo çertos de tal leys expresamente et renunçiamos et partimos de nos et de nosas voses et herdeyros, et renunçiamos eso meesmo et apartamos de nos a outra (sic) qualquer auxilio ordenario ou extraordenario, de que nos en esta parte posamos ajudar, et a dita pena pagada ou non pagada, que todavia esta dita doaçon et as cousas en ela contiudas et declaradas fiquen firmes et vallan para senpre. Et eu, o abbade dom Lourenço Peres et Estevoo Domingues, prior do dito moesteyro de Santa Maria d'Aziveiro et confesus frade (tachado) do dito moesteyro, presentes estavan por sy et en nome dos ditos moesteyros et conventos et do dito cabidoo et mesa capitolar et personas et coengos da dita iglesia, asy o reçeberon.

Esto foy et pasou a porta prinçipal da iglesia de San Johan de Lageas, dia, mes et annos susoditos.

Testemoyas ( $\mathrm{sic}$ ) que presentes estavan: Rodrigo Yanes, clerigo, rector da iglesia de San Johan de Lagees, et Gonçalvo Pedreyro, et Fernan de Magos, et Estevoo Folloyro, et Gonçalvo Fernandes, do Pumar, moradores en a dita fregisia de Lagees, et outros.

Et eu, Gonçalvo Fernandes, notario del rey en terra d'Orzellon et de Doçon, a esto presente foy et escripvi, et meu signo fis, que tal he.

Et non enpeesca onde vay sobre escripto en huun lugar que dis annos et en outro lugar que dis forma et en este outro lugar dela, que asy han de dizer. 
1401, mayo, 19.- San Martín de Cameixa.

(Mato)

Testamento de Estevo Pérez, clérigo, el cual dona tres partes iguales de todos los bienes que posee en el lugar do Outeiro, parroquia de San Martiño de Cameixa, a los monasterios de Santa María de Aciveiro y Santa María de Oseira, y al cabildo y mesa capitular de la Catedral de Santiago.

Inserto en fols. 39r.- 39v de SANTIAGO, AC, Índice General de Legajos, leg. 5 (Tumbo D), de fecha 1447, julio, 28 , fols. 38r-40r, copia simple.

En o nome de Deus, amen. Anno do nasçemento de noso Sennor Jhesu Christo de mill et quatroçentos et huun annos, sabado, des et nove dias do mes de mayo. Sabean todos quantos este testamento vire $<\mathrm{n}>$ como Estevoo Peres, clerigo, morador en o Outeyro, fregisia de San Martino de Cameija, seendo saao en o corpo et en a voontade, con todo meu siso et entendemento que Deus tevo por ben de mandar, et temendo de morte morte (sic) que he cousa natural et por que todos avemos de pasar, et por tal que depois da mina morte non venna contenda ontre algunos sobre meus beens, faço et ordeno de min et de meus beens mia manda et meu testamento et mina postromeyra voontade valedeyro para senpre. Iten mando primeyramente a mina alma \doo/ a (tachado) meu Sennor Jhesu Christo, que a conprou et remio por lo seu Santo Sange, justo, priçioso; et rogelle moyto umildosamente que me queira perdoar os males et errores que contra a sua voontade fije, et rogo por merçede a a Virgen Santa Maria, sua Madre, que seja mina abogada en a ora da morte et a o die do juiso, et lle roge que me queira perdoar. Iten mando que se se acontesçer de eu morrer en a çidade de Santiago ou en o moesteyro de Santa Maria d'Azeveyro ou en o moesteyro de Santa Maria d'Oseira, que enterren o meu corpo en cada huun dos ditos lugares; et mando que se me eu finar a quatro legoas da fregisia de San Martino de Cameija, que o meu yrel seja tiudo de yr por lo meu corpo et trajerlo a a dita iglesia de Cameyja; et mando que se me eu finar mays lonje et onde Deus quiser, que meu yrel seja tiudo, depoes que as minas carnes foren despesas, de yr por los meus osos et trajerlos et sepultarlos en o adro da dita iglesia de Cameyja, ali onde jas meu padre Pedro Martines, do Outeyro. Item mando a a dita eglesia en dous annos huun moyo de de vino, et huun destes puçaas que seja para a obra da dita iglesia; item mando a iglesia de Santa Maria de Jovencos huun puçal de vinno en tres annos; item mando a iglesia de San Fiins de Brues huun sesto de vinno ou oyto maravedis; item mando a iglesia d'Alvarellos huun puçal de vinno en dous annos; item mando a San Domingo de Ribadavea en dous annos tres sesteyros de vinno lou/ 
quinse maravedis; item mando a San Lazaro oyto maravedis, et que llos den logo; item mando a San Johan de Lageas seys maravedis; item mando para sacar cativos de terra de mouros tres maravedis; item mando a o clerigo ou clerigos que me toveren por la maao et me soltaren quando eu finar des maravedis. Item mando que o dia $/$ Fol. 39v. que me enterraren, que me digan doze misas et que dean a cada clerigo quatro maravedis et que me digan dous salteyros o qual dia que me enterraren et en outro dia; item mando que me digan dous triintanarios de misas de sacrifiçio en dous annos por la mia alma et de aqueles que eu soo tiudo; item mando que todoslos meus beens raizes et casas que eu ajo en o lugar do Outeyro et en toda a fregisia de San Martino de Cameyja, que a min perteesçen por parte de meu padre et madre, asy de conpras como de gaanças et eu gaançar ata tenpo de mina morte, mando huun terço deles a o moesteyro de Santa Maria d'Aziveyro et convento entregamente, et ho outro terço dos ditos beens mando a o moesteyro et convento de Santa Maria d'Oseira entregamente, et mando o outro terço dos ditos beens entregamente a o cabidoo et coengos et mesa capitolar da iglesia et see cathedral de Santiago. Et eles et os ditos moesteyros sejan tiudos de rogar a Deus por min et por aqueles que eu soon tiudo, et outorgo et dou por firme et estavele para todo senpre huna doaçon, que eu, o dito Estevoo Peres, min et meu Ipadre/ Pedro Martines avemos feyta a os sobreditos moesteyros et cabidoo. Et mando que todos los outros beens moveles et raises que mandados et doados non son, ondequer que vos aja a tenpo de mina morte, a Pedro Pedreyro, meu sobrinno, fillo de Gonçalvo Pedreyro, de Lajeas, et de mina cormaa Sancha Fernandes, que os aja por jur de herdade para senpre et el que cumpla minas mandas et meu testamento et faça ben por mina alma et d'aqueles que eu soo tyudo. Et mando que me façan huna oferca ( sic) en a iglesia de Cameyja, por dia de Todolos Santos, de pan et vinno et carne ou pescado como mellor poder segundo uso et custume. Et faço a o dito Pedro Pedreyro yrel et conpridor en todos meus beens segundo dito he. Et rogo a Gonçal do Barro, clerigo, morador en Cameyja, que ajude a conprir estas minas mandas et testamento por lo meu et sen seu danno, et mandolle por lo afan que comigo levar viinte maravedis, et aparto todos los outros meus parentes et parentas en huun castineyro que esta en o dito lugar do Outeyro, tras lo çeleyro de Fernan Garrusin, et mays en çinquo soldos de boa moeda, et mays de meus beens non se atendan. Et esta dou et outorgo por mina manda et testamento et codiçillo et postromeyra voontade, et quito et outorgo qualquer da mina parte ou da estraya que contra esta mina manda et testamento et codiçillo et postromeyra voontade vier, for ou pasar, que aja a mina maldiçon et a yra de Deus, et demays que peyte por pena a este meu yrel et conpridor mill maravedis, et a el rey outro tanto peyte; et a pena pagada ou non, todavia esta mina manda et cousas en ela contiudas fiquen firmes et vallan para senpre. 
Feyta et outorgada a dita manda (tachado) en o dito lugar do Outeyro, onde mora o dito Estevoo Peres, clerigo.

Testemoyas (sic) que a esto foron presentes et rogadas por parte do dito Estevoo Peres: Lourenço Eanes, de San Christovo, et Lourenço Vidal, de Pareda, et Lourenço Calado, et Johan Tourinno, et Pedro Vidal, moradores en a dita fregisia de Cameyja.

Et eu, Pascoal Fernandes, notario publico del rey en terra de Orzellon et de Doçon, a esto presente foy et escripvi et meu nome et signal aqui puje en testemoya de verdade que tal the/.

Et non enpeesca onde vay sobre raydo (tachado) escripto ontre rengloos onde dis "moesteyro" et en outro et en outro (sic) lugar hu diz min lugar onde dis (sic), en que eu, notario, o salvo.

\section{8}

1406, [...], [...].- Oseira.

(Lalín y Deza)

El abad Alonso concede en foro a Afonso Fernández, de Goyans, las heredades de Beiláns, en la parroquia de San Miguel de Goyáns, por pago de una tega de pan, más otras prestaciones.

ORENSE, AC, n. ${ }^{\circ} 4677$, no localizado en el archivo.- Extr., Repertorio, pág. 98.

Catálogo: LeIrós, Catálogo, n. ${ }^{\circ} 4677$.

\section{9}

$[1427]^{*}$

(Aguada)

El abad Gómez afora a Rodrigo Alfayate y a su mujer Inés Rodríguez, los lugares en las Lamas de Aguada, que se dicen Brandián de Cima y Brandián de Fondo, un molino y una venta que tenía María Amada, por tres cuarteros de pan, otros servicios y vasallaje.

ORENSE, AC, extr., Repertorio, pág. 34.

* Observaciones: El Repertorio lo fecha en 1437, cuyo año no coincide con el abad Gómez; necesariamente debe ser 1427 . 


\section{ÍNDICE ONOMÁSTICO}

\section{Advertencia al índice onomástico}

Se encuentran referenciadas todas las personas que se mencionan en la documentación.

El número o números que siguen a cada nombre indican el documento o documentos en el que se encuentra.

El cargo, profesión o filiación que posee una persona se escribe con la grafía actual, para ofrecer mayor claridad y accesibilidad, excepto en aquellas palabras que sólo en su forma arcaica conservan su total significación, como por ejemplo "mestre".

Los nombres escritos entre paréntesis mencionan el utilizado en la historiografía, para su mejor localización: "[Alfonso IX]".

Affonso Viviançe, vicario del obispo de Orense, 3 .

Afonso Fernández, de Goyans, 8.

Afonso Nunez, escudero de Castrelo, 4.

Afonso, frey, abad de Oseira, 4.

Afonso, frey, tesorero de Oseira, 3.

Alfonso, rey dom, [posiblemente Alfonso XI], 4.

Alonso, abad de Oseira, 8.

Bonifacius, episcopus, [Papa Bonifacio VIII], 2.

Diego, frey, monje de Oseira, 1.

Domingo [...], 5 .

Domingo Martinez, notario público, 3 .

Domingo Perez, 1.

Domingo, frey, abad de Oseira, 5.

Esteevo/Estevoo Peres, clérigo, morador en Outeiro de San Martiño de Cameixa, hijo de Pedro Martines, do Outeyro, 6, 7.

Estevo de San Lourenço, frey, 1.

Estevoo Domingues, prior de Santa María de Aciveiro, 6.

Estevoo Folloyro, morador en San Juan da Laxe, 6.

Fernan de Magos, morador en San Juan da Laxe, 6.

Fernan Garrusin, 7.

Fernando d’Asma, frey, monje de Oseira, 5.
Françisco Peres, notario del rey en Castela, Búval y Bolo de Senda, 1.

Garçia, frey, mestre de Oseira, 3.

Gillelme, frey, enfermero de Oseira, 3.

Gómez, abad de Oseira, 9.

Gonçal do Barro, clérigo, morador en Cameixa, 7.

Gonçalo Gil de Llamas, escudero de Castrelo, 4.

Gonçalo, señor don, obispo de Orense, 3 .

Gonçalvo Fernandes, do Pumar, morador en San Juan da Laxe, 6.

Gonçalvo Fernandes, notario del rey en terra de Orcellón y Dozón, 6.

Gonçalvo Pedreyro, de Lajeas, morador en San Juan da Laxe, marido de Sancha Fernandes, padre de Pedro Pedreyro, 6, 7.

Johan Ares, marido de Maria Eanes, 3.

Johan de Deus, 5.

Johan Fernandez, notario por Domingo Martinez, 3.

Johan Gomez, marido de Maria Vaasquez, 1.

Johan Lopez, 1.

Johan Martines, home del abad frey Pedro de Oseira, 3.

Johan Martinez, de Barreo de Villamarin, 3.

Johan Peres, 1. 
Johan Perez de Novoa, 4.

Johan Perez, da Regeira, 1.

Johan Perez, de Piellas, 3.

Johan Perez, escudero de Villamarín, 3.

Johan Tourinno, morador en San Martiño de Cameixa, 7.

Johan, fray, monje de Oseira, 1.

Lourenço Calado, morador en San Martiño de Cameixa, 7.

Lourenço Eanes, de San Christovo, morador en San Martiño de Cameixa, 7.

Lourenço Peres, dom, abad de Santa María de Aciveiro, 6.

Lourenço Vidal, de Pareda, morador en San Martiño de Cameixa, 7.

Lourenço, frey, estaleiro de Oseira, 3.

Lourenço, frey, prior de Oseira, 3.

María Amada, 9.

Maria Eanes/Anes, mujer de Johan Ares, 3.

Maria Fernandez Coronel, doña, 3.

Maria Vaasquez, mujer de Johan Gomez, 1.

Martin Monteyro, da enfermaria, 3.

Migell, don frey, abad de Oseira, 1.

Migell, frey, monje de Oseira, 1.

Pascoal Fernandes, notario público en terra de Orcellón y de Dozón, 7.
Pedro Domingues, dito Foreyro, 3.

Pedro Martines, do Outeyro, morador en San Martiño de Cameixa, padre de Estevoo Peres, 6, 7.

Pedro Pedreyro, hijo de Gonçalvo Pedreyro, de Lajeas, y de Sancha Fernandes, 7.

Pedro Vidal, morador en San Martiño de Cameixa, 7.

Pedro, don frey, abad de Oseira, 3.

Per'Anes, home del abad frey Alonso de Oseira, 4.

Per'Anes, marido de Tareysa Perez, 4.

Roberti, presbítero cardenal de Santa Prudencia, 2.

Rodrig'Alvarez, escudero de Castrelo, 4.

Rodrigo Alfayate, marido de Inés Rodríguez, 9.

Rodrigo Yanes, clérigo, rector de San Juan da Laxe, 6.

Rodrigo, frey, cillerero de Oseira, 3.

Roy F., de Faro, 1.

Sancha Fernandes, mujer de Gonçalvo Pedreyro, de Lajeas, madre de Pedro Pedreyro, 7.

Sancho Garçia, de Sever, 4.

Stevo Perez, 3.

Tareysa Perez, mujer de Per'Anes, 4. 


\section{ÍNDICE TOPONÍMICO}

\section{Advertencia al índice toponímico}

Este índice está escrito en lengua gallega, pues el Nomenclátor de Galicia ${ }^{6}$ elaborado por los dictámenes de la Comisión de Toponimia y las normas dictadas por la Xunta de Galicia sólo contemplan como forma oficial la toponimia en dicha lengua, ignorando otras denominaciones establecidas en el idioma español.

Tanto los nombres oficiales actuales como sus variantes antiguas o no oficiales se ofrecen por orden alfabético.

El número o números que siguen a cada nombre indican el documento o documentos en el que se encuentra.

Los nombres oficiales actuales van en versalita; si el nombre antiguo coincide con la denominación oficial actual, se remite a ella. Así por ejemplo "Brues" se remite a BRUÉs, nombre oficial actual de esa parroquia.

Los nombres que van en cursiva son topónimos localizados documentalmente, pero que no se localizan en la actualidad, como por ejemplo Brandián de Cima, lugar de.

Las variantes antiguas $-\mathrm{y}$ las no oficiales hoy día-, se remiten siempre a su equivalente actual, salvo las que no pudieron localizarse. A continuación del nombre oficial, entre paréntesis, van todas las variantes gráficas de ese topónimo citadas en la documentación, y después de su localización, los números de todos los docs. en los que el topónimo se cita.

Tanto los apellidos de personas que son topónimos como los topónimos propiamente dichos sólo son localizados e identificados en aquellos casos que, o bien por los datos facilitados en los documentos donde aparecen, o por ser única la denominación existente en la zona geográfica a la que se refiere, hace precisa su ubicación.

La información que se ofrece entre corchetes "[ ] ] indica que en la documentación existe una mención jurisdiccional/histórica del topónimo al que se refiere. Ej.: [En terra de Orcellón].

Aciveiro, Santa María de.- (Azeveyro, Aziveyro,

Santa Maria d'Aziveyro).- Parroquia, antigo mosteiro benedictino.- Prov. de Pontevedra. Concello de Forcarei.- 6, 7.

Albarellos, San Miguel de.- (iglesia d'Alvare-

llos).- Parroquia.- Prov. de Ourense. Conce-

llo de Boborás.- 7.

Alquirime, tulla d', 3, v. BelquiRIME.

Alvarellos, iglesia d', 7, v. Albarellos, San Miguel de.

Aragon, qunno d', 6, v. ArAGón.

Aragón.- (Aragon).- Antigo reino hispánico; hoxe, rexión histórica de España; título de soberanía da Coroa de España.- 6.
Asma, frey Fernando d’, 5, v. Asma.

Asma.- (Asma).- Pequena comarca natural ou val ó redor do río Asma, que se situa entre os concellos de Chantada e Carballedo, prov. de Lugo.- 5.

Azeveyro, mosteiro de Santa Maria d', 7, v. ACIVEIRO.

Aziveyro, mosteiro de Santa Maria d’, 7, v. ACIVEIRO.

Aziveyro, Santa Maria d’, 6, v. Aciveiro.

BAILÁs, lugar de.- (Beilans).- Parroquia de San Miguel de Goiáns. Prov. de Pontevedra. Concello de Lalín.- 8.

${ }^{6}$ Nomenclátor de Galicia. Toponimia oficial das provincias, concellos, parroquias e lugares, [CD ROM], [s.1.], Xunta de Galicia, [d.1. 2004]. 
Barreo de Villamarin, Johan Martinez de, 3, v. BARRIO, O.

Barreo, cassar de, 3, v. BARRIO, O.

Barreo, lugar de, 3, v. BARRIO, O.

BARRIO, O, lugar de.- (Barreo de Villamarin, Barreo).- Parroquia de Santiago de Vilamarín. Prov. de Ourense. Concello de Vilamarín.- 3.

Barro, Gonçal do, 7.

Beilans, heredades de, 8, v. BAILÁs.

Belquirime, lugar de.- (Alquirime).- Parroquia de San Vicente de Reádegos. Prov. de Ourense. Concello de Vilamarín.

Bidoedo, villa de, 1, v. BIDUEDO.

Biduedo, lugar de.- (Bidoedo).- Parroquia de Santra Baia de Pereda. Prov. de Ourense. Concello de San Cristovo de Cea.- 1.

Bollo de Senda, 1.- Antiga terra xurisdiccional sita no noroeste da prov. de Ourense.

Brandián de Cima, lugar de, 9 .- Lugar das Lamas. Parroquia de San Baia de Aguada. Prov. de Lugo. Concello de Carballedo.

Brandián de Fondo, lugar de, 9.- Lugar das Lamas. Parroquia de San Baia da Aguada. Prov. de Lugo. Concello de Carballedo.

Brues, San Fiins de, 7, v. BruÉs, San Fiz de.

BRuÉs, San Fiz de.- (San Fiins de Brues).- Parroquia. Prov. de Ourense. Concello de Boborás.- 7.

BúBAL.- (Buval).- Pequena comarca natural ou val ó redor do río Búbal, que se situa no concello de Carballedo, prov. de Lugo.- 1.

Buval, 1, v. BúBAL.

Cameija, San Martino de, 6, 7, v. CameIXA, San Martiño de.

CAmeixA, San Martiño de.- (San Martino de Cameija, iglesia de Cameyja, San Martinno de Cameyja).- Parroquia.- [En terra de Orcellón].Prov. de Ourense. Concello de Boborás.- 6, 7.
Cameyja, iglesia de, 7, v. CAMEIXA, San Martiño de.

Cameyja, San Martinno de, 6, v. CameIXa, San Martiño de.

Castella, 1.- Antiga terra xurisdiccional sita na prov. de Ourense.

Castrello, escuderos de, 4, v. Castrelo, San Cibrao.

CAstrelo, San Cibrao.- (Castrello).- Parroquia.Prov. de Ourense. Concello de San Cristovo de Cea.- 4.

Doçon, terra de, 6, 7.- Antiga terra xurisdiccional sita na prov. de Pontevedra, ó redor dos actuais concellos de Lalín e Dozón.

Faro, Roy F. de, 1.

Figeyredo, lugar de, 1.

Fondo DE VILA, lugar de.- (Fondo de Villa).Parroquia de Santiago de Vilamarín. Prov. de Ourense. Concello de Vilamarín.- 1, 3.

Fondo de Villa, lugar de, 1, 3, v. Fondo DE VILA.

Goláns, San Miguel de.- (Goyans, San Miguel de Goyans).- Parroquia.- Prov. de Pontevedra. Concello de Lalín.- 8.

Goyans, Afonso Fernández de, 8, v. Goiáns, San Miguel de.

Goyans, San Miguel de, 8, v. Goláns, San Miguel de.

Jovencos, Santa Maria de, 7, v. Xuvencos, Santa María de.

Lageas, San Johan de, 7, v. LAXAs, San Xoán de.

Lagees, iglesia de San Johan de, 6, v. LAXAs, San Xoán de.

Lajeas, Gonçalvo Pedreyro, de, 7, v. LAXAs, San Xoán de.

Lamas de Aguada, lugares de, 9, v. LAmAs, As.

Lamas, As, lugar de.- (Lamas de Aguada).Parroquia de Santa Baia de Aguada. Prov. de Lugo. Concello de Carballedo.- 9. 
Laterani, 2, v. LETRÁN.

LaXas, San Xoán de.- (San Johan de Lageas, iglesia de San Johan de Lagees, Lajeas).Parroquia.- Prov. de Ourense. Concello de Boborás.- $6,7$.

LETRÁN.- Lugar da cidade de Roma, actualmente pertencente á Cidade do Vaticano, onde se atopa a Basílica de San Xoán de Letrán.2.

Llamas, Gonçalo Gil de, 4.

Magos, Fernan de, 6.

Malburgo, casar de, 4.- Parroquia de Santiago de Vilamarín. Prov. de Ourense. Concello de Vilamarín.

Novoa, Johan Perez de, 4, v. SANto Estevo DE NóvoA, Santo Estevo

Orzellon, terra d', 6, 7.- Antiga terra xurisdiccional do noroeste da prov. de Ourense.

Ourense, bispo d', 3, v. OURENSE.

OuRENSE.- (Ourense).- Cidade, bispado, concello e prov. de Ourense.- 3.

Outeiro, O, lugar de.- (do Outeyro, o Outeyro).Parroquia de San Martiño de Cameixa. Prov. de Ourense. Concello de Boborás.- 6, 7.

Outeyro, lugar do, 6, 7, v. Outeiro, O.

Outeyro, o, 7, v. OuteIro, O.

Outeyro, Pedro Martines do, 6, 7, v. OuteIro, O.

Pareda, Lourenço Vidal, de, 7, v. PeredA.

Pereda, lugar de.- (Pareda).- Parroquia de Santa Baia de Pereda. Prov. de Ourense. Concello de San Cristovo de Cea, 7.

Pielas, lugar de.- (Piellas).- Parroquia de Santa María a Real de Oseira. Prov. de Ourense. Prov. de Ourense.- 3.

Piellas, Johan Perez, de, 3, v. Pielas.

Pumar, Gonçalvo Fernandes do, 6.

Regeira, Johan Perez da, 1, v. REgueIRA GRANDE, A.
Regueira Grande, A, lugar de.- (da Regeira).Parroquia de Santiago de Vilamarín. Prov. de Ourense. Concello de Vilamarín.- 1.

Ribadavea, San Domingo de, 7, v. RibadaviA, San Domingos de.

Ribadavia, San Domingos de.- (San Domingo de Ribadavea).- Parroquia e antigo mosteiro dominico.- Prov. de Ourense. Concello de Ribadavia.- 7.

San Christovo, Lourenço Eanes de, 7, v. SAN CRIstovo.

San Cristovo, lugar de.- (San Christovo).Parroquia de San Martiño de Cameixa. Prov. de Ourense. Concello de Boborás.- 7.

San Lourenço, frey Estevo de, 1.

Sancte Pudenciae, 2, v. Santa Prudencia.

Santa Prudencia.- Basílica sita na cidade de Roma.

SAntiago de Compostela.- (Santiago).- Cidade, arcebispado e concello.- Prov. da Coruña. Concello de Santiago de Compostela.- 6, 7.

Santiago, cathedral de, 6, v. SANTIAGo DE ComPOSTELA.

Santiago, cidade de, 7, v. Santiago de ComposTELA.

Santiago, iglesia catedral de, 6, 7, v. SAntiago de Compostela.

Santo Estevo de Nóvoa, Santo Estevo.(Novoa).- Parroquia.- Prov. de Ourense. Concello de Carballeda de Avia.- 4.

Sever, lugar de.- (Sever).- Parroquia de Santa Baia de Aguada. Prov. de Lugo. Concello de Carballedo.- 4.

Sever, Sancho Garçia de, 4, v. SEvEr.

Toubes, lugar de.- (villa de Touges, Touves).Parroquia de San Román de Viña. Prov. de Ourense. Concello de San Cristovo de Cea.3,4 .

Touges, villa de, 3 , v. ToubEs. 
Touves, tulla de, 4 , v. ToubEs.

Vilamarín, lugar de.- (villa de Villamarin).Parroquia de Santiago de Vilamarín. Prov. de Ourense. Concello de Vilamarín.- 3.

Vilamarín, Santiago de.- (Santiago de Villamarim, Villamarin, Villamarin).- Parroquia.- Prov. de Ourense. Concello de Vilamarín.- 1, 3, 4.

Villamarim, Santiago de, 4, v. Vilamarín, Santiago de.
Villamarin, 1, v. Vilamarín, Santiago de.

Villamarin, escudero de, 3, v. Vilamarín, Santiago de.

Villamarin, villa de, 3, v. VilamaRín, lugar de.

Xuvencos, Santa María de.- (Santa Maria de Jovencos).- Parroquia.- Prov. de Ourense. Concello de Boborás.- 7. 